\title{
Clinical and Computed Tomography
}

\section{Characteristics for Early Diagnosis of Peripheral Small-cell Lung Cancer}

\author{
Xiaochuan Zhang ${ }^{1,2, *}$, Fajin Lv',*, Binjie Fu $\mathbb{D}^{\prime}$, Wangjia Li $\mathbb{D}^{\prime}$, Ruiyu Lin', Zhigang Chu $\mathbb{D}^{\prime}$ \\ 'Department of Radiology, The First Affiliated Hospital of Chongqing Medical University, Chongqing, 4000 I6, People's Republic of China; \\ ${ }^{2}$ Department of Radiology, Chonggang General Hospital, Chongqing, 400080, People's Republic of China \\ *These authors contributed equally to this work \\ Correspondence: Zhigang Chu, Tel +86 I8723032809, Fax +86 23 688II487, Email chuzg08I5@I63.com
}

Purpose: To investigate the clinical and computed tomography (CT) characteristics of peripheral small-cell lung cancer (pSCLC) to improve its early diagnosis.

Patients and Methods: In total, 70, 132, 69, and 95 patients with pathological confirmed nodular $(\leq 3 \mathrm{~cm})$ pSCLC, peripheral nonsmall cell lung cancer (pNSCLC), benign lung tumor (pBLT), and inflammatory lesion (pIL) were enrolled in this study retrospectively. The clinical and CT data of studied patients with different lesions were analyzed and compared by univariate analysis. Multivariate analysis was used to reveal the key features to distinguish pSCLC from pNSCLC, pBLT, and pIL, respectively.

Results: Univariate and multivariate analysis of the clinical and CT characteristics of studied patients indicated that 1) compared with pNSCLC and pIL, vessel convergence, spiculation, and peripheral ground-glass opacity were less common in pSCLC; 2) density homogeneity $(\mathrm{OR}=38.84-120.21, P<0.05)$, bronchial cutoff sign $(\mathrm{OR}=10.00-60.13, P=0.001)$, hilar lymph node enlargement $(\mathrm{OR}$ $=22.81-95.08, P<0.0001)$ (pSCLC vs pNSCLC, pBLT, and pIL), male sex $(\mathrm{OR}=5.53-10.92, P<0.05)$ (pSCLC vs pNSCLC and pBLT), and emphysema $(\mathrm{OR}=36.57-56.63, P<0.05)$ (pSCLC vs pBLT and pIL) were significantly and independently associated with pSCLC. Type I and II bronchial cutoff sign, especially type I, were closely related to pSCLC.

Conclusion: Peripheral solid nodules with homogeneous density, bronchial cutoff sign, hilar lymph node enlargement, but without spiculation or vessel convergence in male patients with emphysema should be highly suspected of pSCLC.

Keywords: small-cell lung carcinoma, non-small cell lung carcinoma, tomography, X-ray computed

\section{Introduction}

Nodule is one of the most common manifestations of pulmonary lesions. Regarding the pathological nature, the benign nodules are various, whereas the malignant ones are mainly non-small cell lung cancer (NSCLC) (80-85\%) and small cell lung cancer (SCLC) (15-20\%). ${ }^{1}$ SCLC is the most common primary pulmonary neuroendocrine malignancy and is characterized by rapid growth and greater propensity for early development of widespread metastases. ${ }^{2}$ Each year, SCLC comprises an estimated 250,000 new cases and at least 200,000 deaths globally. ${ }^{3}$ Patients with SCLC have an exceptionally poor prognosis because $60-70 \%$ of cases exhibit hematogenous metastases at initial diagnosis. ${ }^{4}$ The 5-year survival rate is only approximate $10 \%$ for patients with limited-stage SCLC and less than $1 \%$ for those with extensive-stage disease. ${ }^{5,6}$ Surgical resection for early-stage SCLC is associated with improved survival, and some could be potentially cured by operation and postoperative chemotherapy. ${ }^{7,8}$ Therefore, early diagnosis of SCLC seems to be more valuable, and may be very helpful for improving its prognosis.

The majority (90-95\%) of SCLCs arise from lobar or main bronchi. ${ }^{9}$ Thus, they usually appear as large masses that are centrally located within the lung parenchyma or mediastinal masses, which involve at least one hilum. ${ }^{10}$ Both of these have been studied in detail. ${ }^{11-14}$ In a minority $(<5 \%)$ of patients, SCLCs are manifested as peripheral nodules without associated 
lymph node enlargement; ${ }^{15}$ to this date, these have not been well understood. A previous study revealed the computed tomography (CT) features of peripheral SCLCs (pSCLCs) and peripheral NSCLCs (pNSCLCs) were different, and the former were usually manifested as rounded or lobulated nodules with homogeneous density and smooth margin, but without significant spiculation, pleural indentation, or internal necrosis. ${ }^{11,16,17}$ However, these pSCLC features were similar to those of benign tumors, while there has been no comparative study between pSCLCs and peripheral benign lung tumor (pBLTs) or peripheral inflammatory lesions (pILs). Additionally, the sample sizes of pSCLCs in previous studies were relatively small, ${ }^{8,16}$ and larger ones $(>3 \mathrm{~cm})$ were also included. ${ }^{18}$ The CT manifestations of larger pSCLCs may be different from those of smaller ones. These shortcomings introduce a lack of confidence in the early diagnosis of pSCLCs, especially the smaller ones. Thus, the study and comparison of the small-sized pSCLCs with relatively large samples are imperative.

Therefore, it is necessary to further determine the independent clinical and imaging features of pSCLCs for the improvement of its early diagnosis and differential diagnosis. In this study, patients with pathological confirmed nodular pSCLCs, pNSCLCs, pBLTs, and pILs were enrolled retrospectively. Their clinical and CT data were thoroughly studied and compared to reveal the independent features to distinguish pSCLC from other lesions. The results may provide valuable directions for early diagnosis and differential diagnosis of pSCLC.

\section{Methods}

\section{Patients}

This study was approved by the Institutional Review Board of the First Affiliated Hospital of Chongqing Medical University. The requirement for informed consent was waived for this retrospective study. Patients with pSCLC, pNSCLC, pBLT, and pIL confirmed by pathological examination after operation or biopsy were consecutively enrolled in this study. All patients underwent chest CT examinations before operation or therapy. The inclusion criteria were as follows: (1) the lesions were solid, (2) the lesions were located in subsegment or more distal area, (3) the diameters of lesions were less than or equal to $3 \mathrm{~cm}$. Patients were excluded if CT images were of poor quality resulting in the morphological features of nodules could not be well evaluated, or nodules had significant calcification or multiple concomitant lesions. Finally, 70, 132, 69, and 95 patients with pSCLC, pNSCLC, pBLT, and pIL were enrolled in the present study between July 2012 to March 2021. In 132 cases of pNSCLC, there were 113 (85.6\%) adenocarcinomas, 17 $(12.9 \%)$ squamous cell carcinomas, $2(1.5 \%)$ adenosquamous carcinomas; in 69 cases of pBLT, there were $40(58 \%)$ pulmonary hamartomas, $28(40.6 \%)$ sclerosing pneumocytomas or pulmonary sclerosing hemangioma, $1(1.4 \%)$ pulmonary cavernous hemangioma; in 95 cases of pIL, there were $83(87.4 \%)$ nonspecific inflammations, $8(8.4 \%)$ tuberculosis, 4 (4.2\%) fungus.

\section{CT Protocol}

All patients were examined with the use of a 64-slice spiral CT scanner (SOMATOM Definition Flash, Siemens, Germany) with the following settings: tube voltage, $140 \mathrm{kVp}$; tube current calculated according to individual's weight, height, and body mass index; rotation time, $0.5 \mathrm{~s}$; pitch, 1.0 ; collimation, $0.6 \mathrm{~mm}$; slice thickness and interval for axial images, $5 \mathrm{~mm}$ and $5 \mathrm{~mm}$, respectively; and reorganization interval, $1 \mathrm{~mm}$. Upon CT examination, patients were placed in the supine position with both hands near the head. Image acquisition was performed from the level of the thoracic inlet to a level inferior to the costophrenic angle. All patients underwent plain CT scanning and 264 patients (52 with pSCLCs, 98 with pNSCLCs, 69 with pBLTs, and 95 with pILs) underwent contrast enhanced CT scans with a total of 80-100 mL of nonionic iodinated contrast material (Iopamidol, $320 \mathrm{mg} / \mathrm{mL}$, Shanghai Bracco Sine Pharmaceutical Co., Ltd., China) at an injection rate of $3.0 \mathrm{~mL} / \mathrm{s}$; followed by $50 \mathrm{~mL}$ of saline solution via a power injector. Images were obtained with mediastinal (width, 350-400 Hounsfield units (HU); level, 20-40 HU) and lung (width, 1200-1600 HU; level, -500 to $-700 \mathrm{HU})$ window settings.

\section{Clinical Data and Image Analysis}

The patients' clinical data were recorded using the Electronic Medical Record System (Winning Health, China). Clinical data, including the patient's gender, mean age, smoking history, family history of lung cancer, respiratory symptoms, and 


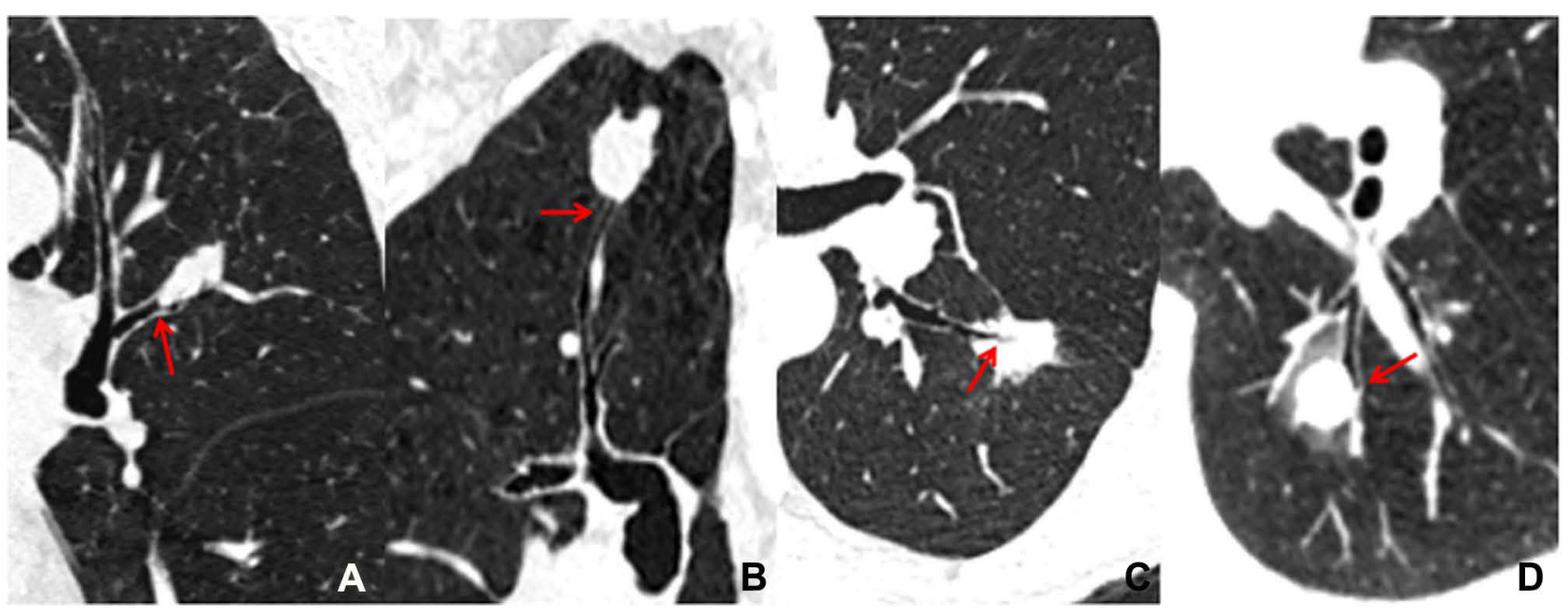

Figure I Four types of bronchial cutoff signs. (A) Type l: the lesion grows along the outline of the bronchus with a noted truncation of the proximal lumen (red arrow). (B) Type II: bronchus (red arrow) is obstructed abruptly by the lesion. (C) Type III: bronchus (red arrow) penetrates into the lesion with tapered narrowing and interruption. (D) Type IV: bronchus (red arrow) runs around the periphery of the lesion and is displaced, compressed, and narrowed by it.

tumor biomarkers, including cytokeratin 19 fragment antigen 21-1 (Cyfra 21-1), progastrin-releasing peptide (proGRP), squamous cell carcinoma antigen (SCC-Ag), carcinoembryonic antigen (CEA), and neuron-specific enolase (NSE) were recorded. All clinical data were collected by the radiologists through the Electronic Medical Record System.

CT data were independently reviewed on a picture archiving and communication system (PACS) workstation (Carestream Vue PACS). CT images of all patients were reviewed by two radiologists (with 15 and 10 years of experience in chest CT, respectively) who were blinded to the clinical data and results. Data analysis was based on both the original and the reformatted images. When the interpretations of the two radiologists differed, the discrepancies were resolved by consensus.

The following markers were evaluated on the CT images: nodule size (the mean of the longest diameter and the perpendicular diameter on axial CT images), location (upper, lower or middle lobes), shape (round, oval, or irregular), density homogeneity (yes/no), CT value on plain CT scan, homogeneous enhancement (yes/no), $\Delta \mathrm{CT}$ values ( $\Delta \mathrm{CT}$ value = peak $\mathrm{CT}$ value on contrast enhanced CT scan $-\mathrm{CT}$ value on plain CT scan), vacuole sign (yes/no), smooth margin (yes/ no), spiculation (yes/no), spinous process sign (yes/no), lobulation (yes/no), bronchial cutoff sign (yes/no), peripheral ground glass opacity (GGO) (yes/no), obstructive pneumonia (yes/no), vessel convergence (yes/no), pleural indentation (yes/no), nodule-lung interface (well-defined or ill-defined), hilar lymph node enlargement (yes/no), mediastinal lymph node enlargement (yes/no), emphysema (yes/no). Lymph node enlargement refers to the case in which the short diameter of mediastinal or hilar lymph node is $>1 \mathrm{~cm}$. The irregular shapes included fusiform, vermiform, branching, beaded, pine-cone-like shapes or others. Vacuole sign is defined as round or irregular air attenuation with 1-2 mm in diameter in a nodule. ${ }^{19}$ Spinous process sign is defined as nodule edges protruded sharply with the appearance of a small triangle. ${ }^{20}$ Smooth margin is defined as the margin of the nodule is even without any short or long spiculation. ${ }^{21}$ The bronchial cutoff sign refers to the abrupt truncation of a bronchus from obstruction caused by nodule, mucus plugging, trauma or foreign bodies. According to the relationship between nodule and bronchus set by Soichi Akata et al, ${ }^{22}$ the bronchial cutoff sign in this study was classified into four types: type I, the nodule grew along the outline of bronchus with the truncation of the proximal lumen; type II, bronchus was obstructed abruptly by the nodule; type III, bronchus penetrated into the nodule with tapered narrowing and interruption; and type IV, bronchus ran around the periphery of the nodule and was displaced, compressed, and narrowed by nodules (Figure 1).

\section{Statistical Analysis}

The clinical data of the studied patients and CT features of different lesions were statistically analyzed with the software package SPSS (version 22.0, IBM, NY, USA). Continuous variables were expressed as means \pm standard deviations or 
median and range according to whether they were normally distributed or not (Kolmogorov-Smirnov test), and categorical variables were expressed as numbers. To compare differences in variables among different lesions, the Mann-Whitney $U$-test was used for patient ages, nodules sizes, CT values on plain scan, and $\Delta \mathrm{CT}$ values, the Pearson Chi-square test was used for gender, smoking history, and frequencies of different CT features. Variables with statistical differences in univariate analysis were further included in binary logistic regression analysis to determine independent factors for predicting pSCLCs in a stepwise manner, following the Forward LR method in SPSS. ${ }^{23}$ Considering the difference among the pNSCLC, pBLT and pIL, the pSCLC group was analyzed with the pNSCLC, the pBLT, and the pIL group, respectively. The removal of variables was based on likelihood ratio statistics with a probability of 0.1 . Receiver operating characteristic (ROC) curves were used for taking prediction probability and calculating the available area under the curve (AUC), sensitivity, specificity, positive predictive value (PPV), and negative predictive value (NPV). A p-value of $<0.0167$ was considered to be statistically significant after Bonferroni correction. A $P$-value of $<0.05$ was considered to indicate a statistically significant difference in binary logistic regression analysis.

\section{Results}

\section{Clinical Characteristics of Studied Patients}

The clinical characteristics of the studied patients are listed in Table 1. There were a higher proportion of male patients in the pSCLC group than in the pNSCLC and pBLT groups (each $P<0.0167)$. A higher proportion of smokers $(71.4 \%)$ were found in the pSCLC group than in other groups (each $P<0.05$ ). The mean age and symptoms of patients with pSCLC were higher and more common than those of patients with pBLT and pIL (each $P<0.0167$ ). Among those patients with tumor biomarkers, the numbers of cases with abnormal proGRP were higher in the pSCLC group than in other groups (each $P<0.0167$ ); the numbers of cases with NSE were higher in the pSCLC group than in pNSCLC and pIL groups (each $P<0.0167$ ).

\section{Comparison of the CT Features of pSCLC, pNSCLC, pBLT, and pIL}

The CT features of pSCLC, pNSCLC, pBLT and pIL are shown in Table 2. Compared with pBLTs, pSCLCs were larger in size $(P<0.0167)$. Bronchial cutoff sign, density homogeneity, hilar and mediastinal lymph node enlargement, and emphysema were more common in the pSCLC group than in other groups $(P<0.0167)$. Among the 70 pSCLSs, 20 $(28.6 \%)$ were irregular, and $16(80.0 \%)$ of them exhibited fusiform, vermiform, or branching shapes. An unsmooth

Table I Clinical Characteristics of the Studied Patients

\begin{tabular}{|c|c|c|c|c|c|c|c|}
\hline Clinical Characteristics & $\begin{array}{l}\text { pSCLC } \\
(n=70)\end{array}$ & $\begin{array}{l}\text { pNSCLC } \\
(n=132)\end{array}$ & $\begin{array}{l}\text { pBLT } \\
(n=69)\end{array}$ & $\begin{array}{l}\text { pIL } \\
(n=95)\end{array}$ & $P_{1}{ }^{a}$ & $P_{2}{ }^{a}$ & $P_{3}{ }^{a}$ \\
\hline Gender (Male/Female) & $56 / 14$ & $79 / 53$ & $24 / 45$ & $73 / 22$ & 0.004 & $<0.0001$ & 0.627 \\
\hline Mean age (year) & $63.01 \pm 8.00$ & $62.51 \pm 9.78$ & $51.71 \pm 10.74$ & $56.68 \pm 10.70$ & 0.693 & $<0.0001$ & $<0.000$ I \\
\hline Smoking history $(+/-)$ & $50 / 20$ & $70 / 62$ & $|8 / 5|$ & $49 / 46$ & 0.011 & $<0.0001$ & 0.01 \\
\hline Family history of lung cancer (+ / -) & $4 / 66$ & $3 / 129$ & $2 / 67$ & $5 / 90$ & 0.385 & 0.69 & 1 \\
\hline Symptoms (+ / -) & $47 / 23$ & $79 / 53$ & $25 / 44$ & $40 / 55$ & 0.309 & $<0.0001$ & 0.001 \\
\hline Cyfra2I-I (+ / -) & $6 / 9$ & $23 / 40$ & $1 / 10$ & $1 \mathrm{I} / 40$ & 0.801 & 0.178 & 0.272 \\
\hline proGRP (+ / -) & $9 / 4$ & $3 / 42$ & $0 / 8$ & $1 / 34$ & $<0.0001$ & 0.005 & $<0.0001$ \\
\hline SCC-Ag (+ / -) & $4 / 9$ & $4 / 43$ & $\mathrm{I} / 7$ & $2 / 32$ & 0.103 & 0.606 & 0.072 \\
\hline CEA $(+/-)$ & $5 / 19$ & $10 / 43$ & $0 / 14$ & $0 / 50$ & 1 & 0.137 & 0.004 \\
\hline NSE (+ / -) & $11 / 10$ & $5 / 42$ & $1 / 8$ & $3 / 42$ & 0.001 & 0.049 & $<0.0001$ \\
\hline
\end{tabular}

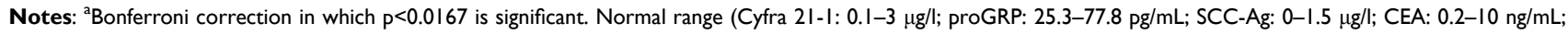
NSE: $0-16.3 \mathrm{ng} / \mathrm{mL}$ ). PI: the P value of PSCLC vs PNSCLC; P2: the P value of PSCLC vs PBLT; P3: the P value of PSCLC vs plL. +: positive or abnormal, $-:$ indicates negative or normal.

Abbreviations: pSCLC, peripheral small cell lung cancer; pNSCLC, peripheral non-small cell lung cancer; pBLT, peripheral benign lung tumor; plL, peripheral inflammatory lesion; Cyfra 2I-I, Cytokeratin 19 fragment antigen 2I-I; proGRP, progastrin-releasing peptide; SCC-Ag, squamous cell carcinoma antigen; CEA, carcinoembryonic antigen; NSE, neuron-specific enolase. 
Table 2 CT Features of Different Nodules

\begin{tabular}{|c|c|c|c|c|c|c|c|}
\hline CT Features & $\begin{array}{l}\text { pSCLC } \\
(n=70)\end{array}$ & $\begin{array}{l}\text { pNSCLC } \\
(n=132)\end{array}$ & $\begin{array}{c}\text { pBLT } \\
(n=69)\end{array}$ & $\begin{array}{c}\text { pIL } \\
(n=95)\end{array}$ & $P_{1}{ }^{a}$ & $P_{2}{ }^{a}$ & $\mathbf{P}_{3}{ }^{\mathrm{a}}$ \\
\hline Size $(\mathrm{cm})$ & $18.8(10.5-27.0)$ & $19.5(10.0-28.0)$ & $15.5(9.0-27.5)$ & $16.0(10.0-29.5)$ & 0.737 & 0.012 & 0.025 \\
\hline Location (upper/lower/middle lobes) & $35 / 30 / 5$ & $85 / 42 / 5$ & $28 / 32 / 9$ & $46 / 44 / 5$ & 0.123 & 0.372 & 0.834 \\
\hline Shape (regular/irregular) & $50 / 20$ & $122 / 10$ & $69 / 0$ & $81 / 14$ & $<0.0001$ & $<0.0001$ & 0.03 \\
\hline Density homogeneity $(+/-)$ & $69 / 1$ & $88 / 44$ & $56 / 11$ & $74 / 21$ & $<0.0001$ & 0.002 & $<0.0001$ \\
\hline $\mathrm{CT}$ value on plain scan $(\mathrm{HU})$ & $19.7 \pm 13.54$ & $18(-186-54)$ & $13(-159-53)$ & $17(-95-76)$ & 0.02 & 0.006 & 0.082 \\
\hline Homogeneous enhancement $(+/-)$ & $48 / 4$ & $82 / 16$ & $61 / 8$ & $78 / 17$ & 0.139 & 0.477 & 0.091 \\
\hline$\Delta \mathrm{CT}$ value $(\mathrm{HU})$ & $27(7-86)$ & $50(39-104)$ & $41(39-104)$ & $35.86 \pm 23.85$ & $<0.0001$ & 0.022 & 0.149 \\
\hline Vacuole sign $(+/-)$ & $2 / 68$ & $9 / 123$ & $0 / 69$ & $|4 / 8|$ & 0.393 & 0.496 & 0.011 \\
\hline Smooth margin $(+/-)$ & $63 / 7$ & $56 / 76$ & $67 / 2$ & $51 / 44$ & $<0.0001$ & 0.175 & $<0.0001$ \\
\hline Spiculation (+ / -) & $3 / 67$ & $74 / 58$ & $2 / 67$ & $44 / 51$ & $<0.0001$ & I & $<0.0001$ \\
\hline Spinous process sign $(+/-)$ & $7 / 63$ & $14 / 118$ & $1 / 68$ & $11 / 84$ & 0.893 & 0.072 & 0.748 \\
\hline Lobulation $(+/-)$ & $34 / 36$ & $81 / 51$ & $28 / 41$ & $44 / 51$ & $0.08 \mathrm{I}$ & 0.343 & 0.774 \\
\hline Bronchial cutoff sign $(+/-)$ & $36 / 34$ & $33 / 99$ & $6 / 63$ & $8 / 87$ & $<0.0001$ & $<0.0001$ & $<0.0001$ \\
\hline Peripheral GGO (+ / -) & $4 / 66$ & $26 / 106$ & $2 / 67$ & $36 / 59$ & 0.008 & 0.69 & $<0.0001$ \\
\hline Obstructive pneumonia (+ / -) & $13 / 57$ & $9 / 123$ & $2 / 67$ & $9 / 86$ & 0.011 & 0.003 & 0.089 \\
\hline Vessel convergence $(+/-)$ & $3 / 67$ & $79 / 53$ & $0 / 69$ & $3 / 92$ & $<0.0001$ & 0.248 & I \\
\hline Pleural indentation (+ / -) & $6 / 64$ & $71 / 61$ & $1 / 68$ & $30 / 65$ & $<0.0001$ & 0.126 & $<0.0001$ \\
\hline Nodule-lung interface (well-defined or ill-defined) & $63 / 7$ & $123 / 9$ & $68 / 1$ & $88 / 7$ & 0.426 & 0.072 & 0.549 \\
\hline Hilar lymph node enlargement $(+/-)$ & $43 / 27$ & $14 / 118$ & $4 / 65$ & $7 / 88$ & $<0.0001$ & $<0.0001$ & $<0.0001$ \\
\hline Mediastinal lymph node enlargement $(+/-)$ & $35 / 35$ & $26 / 106$ & $0 / 69$ & $8 / 87$ & $<0.0001$ & $<0.0001$ & $<0.0001$ \\
\hline Emphysema (+ / -) & $19 / 51$ & $|1 / / 2|$ & $2 / 67$ & $8 / 87$ & $<0.0001$ & $<0.0001$ & 0.001 \\
\hline
\end{tabular}

Notes: ${ }^{2}$ Bonferroni correction in which $p<0.0167$ is significant. Regular: round or oval. $P_{1}$ : the $P$ value of $p S C L C$ vs $p N S C L C ; P_{2}$ : the $P$ value of $p S C L C$ vs $p B L T ; P_{3}$ : the $P$ value of $p S C L C$ vs plL. + : positive or abnormal, $-:$ indicates negative or normal.

Abbreviations: CT, computed tomography; pSCLC, peripheral small cell lung cancer; pNSCLC, peripheral non-small cell lung cancer; pBLT, peripheral benign lung tumor; plL, peripheral inflammatory lesion; HU, Hounsfield units; GGO, ground glass opacity. 
Table 3 Binary Logistic Regression Analysis of the Clinical and CT Characteristics of Studied Patients

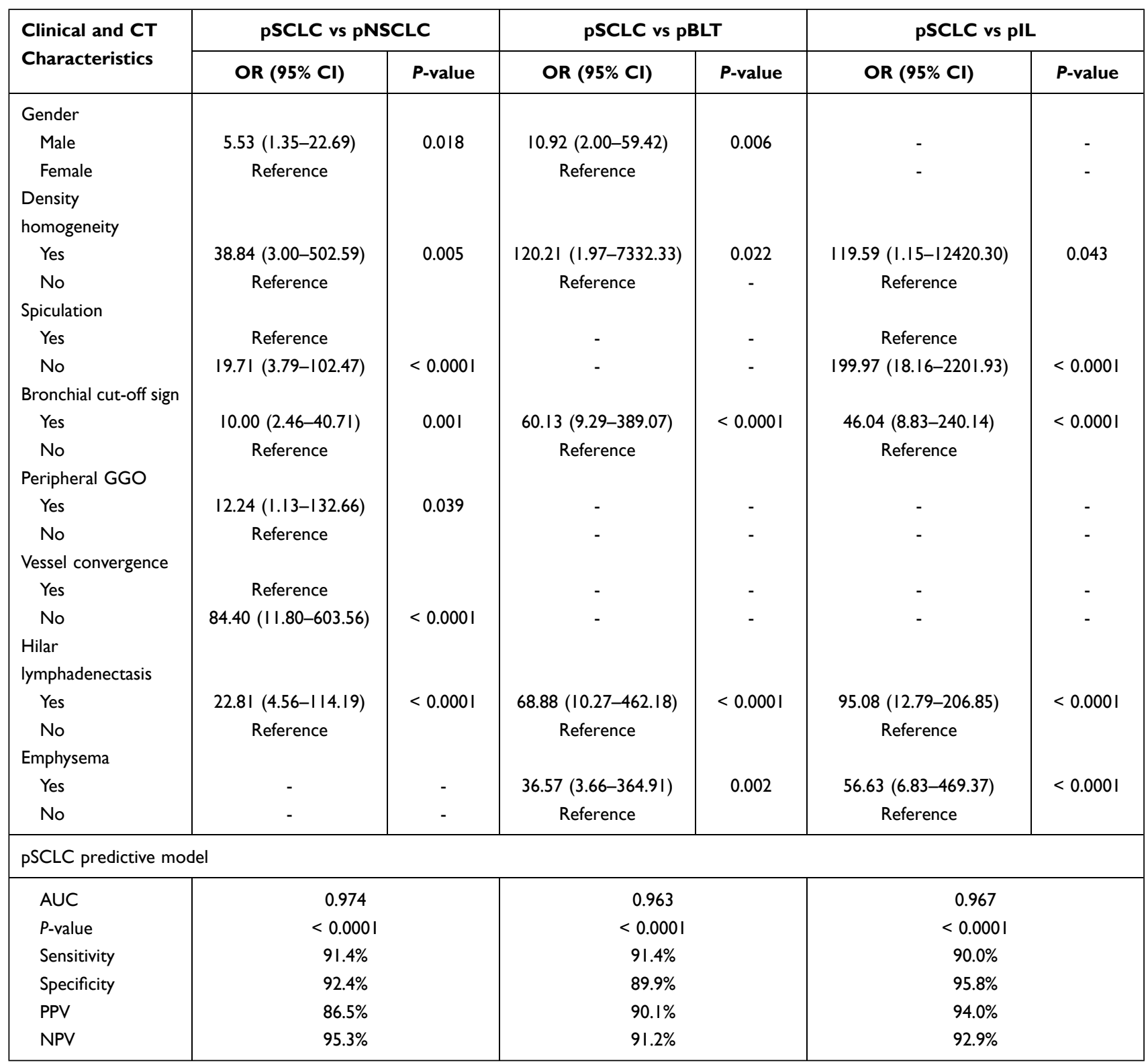

Note: The sign "-" represents that this feature is not an independent predictor between these two groups.

Abbreviations: CT, computed tomography; PSCLC, peripheral small cell lung cancer; pNSCLC, peripheral non-small cell lung cancer; pBLT, peripheral benign lung tumor; plL, peripheral inflammatory lesion; OR, odds ratio; CI, Confidence interval; GGO, ground glass opacity; AUC, area under the curve; PPV, positive predictive value; NPV, negative predictive value.

margin, peripheral GGO, pleural indentation, and spiculation were more common in pNSCLC and pIL groups than in the pSCLC group $(\mathrm{P}<0.0167)$. The mean CT value on plain scan of pSCLC was higher than those of pBLT $(\mathrm{P}<0.0167)$. The $\triangle \mathrm{CT}$ values of pSCLC was lower than those of pNSCLC $(\mathrm{P}<0.0167)$.

Regarding the bronchial cutoff sign, the numbers of type I, II, III, and IV were $6(16.7 \%), 30(83.3 \%), 0,0$ in pSCLC, 0, 13 (39.4\%), 15 (45.5\%), 5 (15.1\%) in pNSCLC, 0, 1 (16.7\%), 0, 5 (83.3\%) in pBLT, and 0, 4 (50\%), $1(12.5 \%), 3$ $(37.5 \%)$ in pIL, respectively. Type II, III, and IV were more common in the pSCLC, pNSCLC, and pBLT groups, respectively (each $P<0.0001$ ), whereas the type I was only detected in the pSCLC group $(P<0.014)$. 


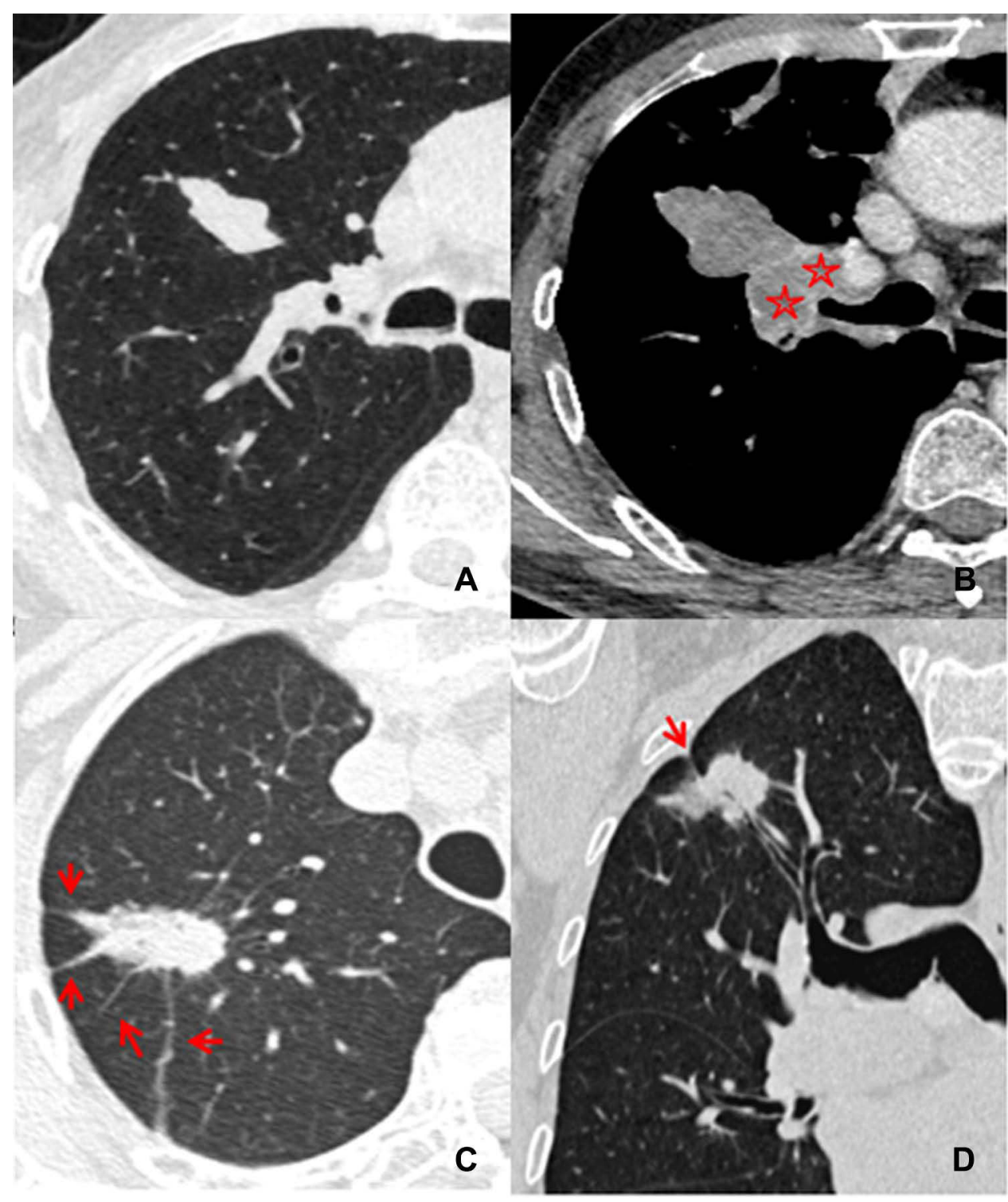

Figure 2 Differentiation between peripheral small-cell lung cancer (pSCLC) and peripheral non-small cell lung cancer (pNSCLC). Axial computed tomography (CT) image in a 76 year old male with pSCLC shows an irregular nodule with homogeneous density and smooth margin (A), 99 days later, the nodule size increases obviously and the right hilar lymphadenectasis is detected simultaneously (red stars) on contrast enhanced CT image (B). Axial and reconstructed CT images in a 73 year old female with pNSCLC show an oval nodule with heterogeneous density, spiculation (red arrows) (C), and pleural indentation (red arrow) (D).

\section{Binary Logistic Regression Analysis of the Clinical and CT Characteristics of Studied Patients}

Table 3 shows the clinical and CT characteristics that independently discriminated pSCLC from pNSCLC, pBLT, and pIL via binary logistic regression analysis. Compared with pNSCLC, male sex, density homogeneity, bronchial cutoff sign, non-vessel convergence, non-spiculation, non-peripheral GGO, and hilar lymph node enlargement were found to be the significantly independent indicators of pSCLC (Figure 2). Also, compared with pBLT, male sex, density homogeneity, bronchial cutoff sign, hilar lymph node enlargement, and emphysema were significantly and independently associated with pSCLC (Figure 3). Additionally, compared with pIL, density homogeneity, bronchial cutoff sign, non-spiculation, hilar lymph node enlargement, and emphysema were found to be the significant and independent indicators of pSCLC (Figure 4). 


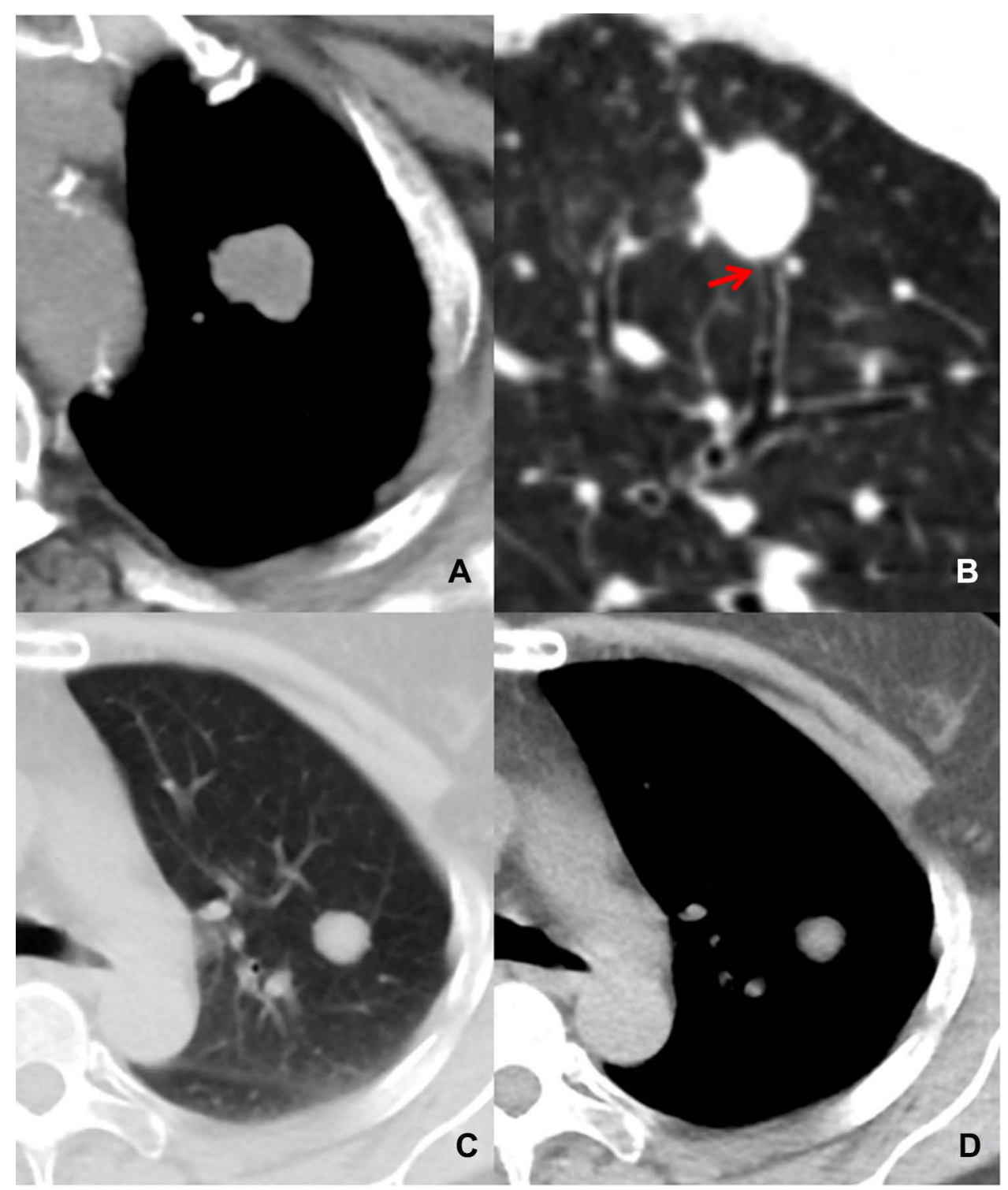

Figure 3 Differentiation between pSCLC and benign lung tumors (pBLT). Axial and reconstructed CT images in a 69 year old female with pSCLC show an oval nodule with a smooth margin, homogeneous density (A) and bronchial cutoff sign (red arrow) (B). Axial CT images in a $5 \mathrm{I}$ year old female with pBLT show a round nodule with smooth margin (C), and homogeneous density (D).

The area under curve (AUC) of the pSCLC predictive model established by the above independent clinical and CT characteristics was 0.974 (sensitivity and specificity, 91.4\% and 92.4\%; PPV and NPV, 86.5\% and 95.3\%) (pSCLC vs pNSCLC), 0.963 (sensitivity and specificity, 91.4\% and 89.9\%; PPV and NPV, 90.1\% and 91.2\%) (pSCLC vs pBLT), and 0.967 (sensitivity and specificity, 90.0\%, and 95.8\%; PPV and NPV, 94.0\%, and 92.9\%), respectively (Figure 5).

\section{Volume Doubling Time of pSCLC}

Among the 70 cases of pSCLCs, 10 (14.3\%) had follow-up CT data before operation or therapy. The calculated median volume doubling time was 78.5 days (range: $37-215$ days).

\section{Discussion}

When a solid nodule is detected on chest CT scan, its possible pathological nature should be evaluated by analyzing morphological features to direct further treatment. The vast majority of the peripheral solid nodules are ILs, NSCLCs, BLTs, and SCLCs. Among them, early determination of the malignancy, especially SCLC, is very important for their 


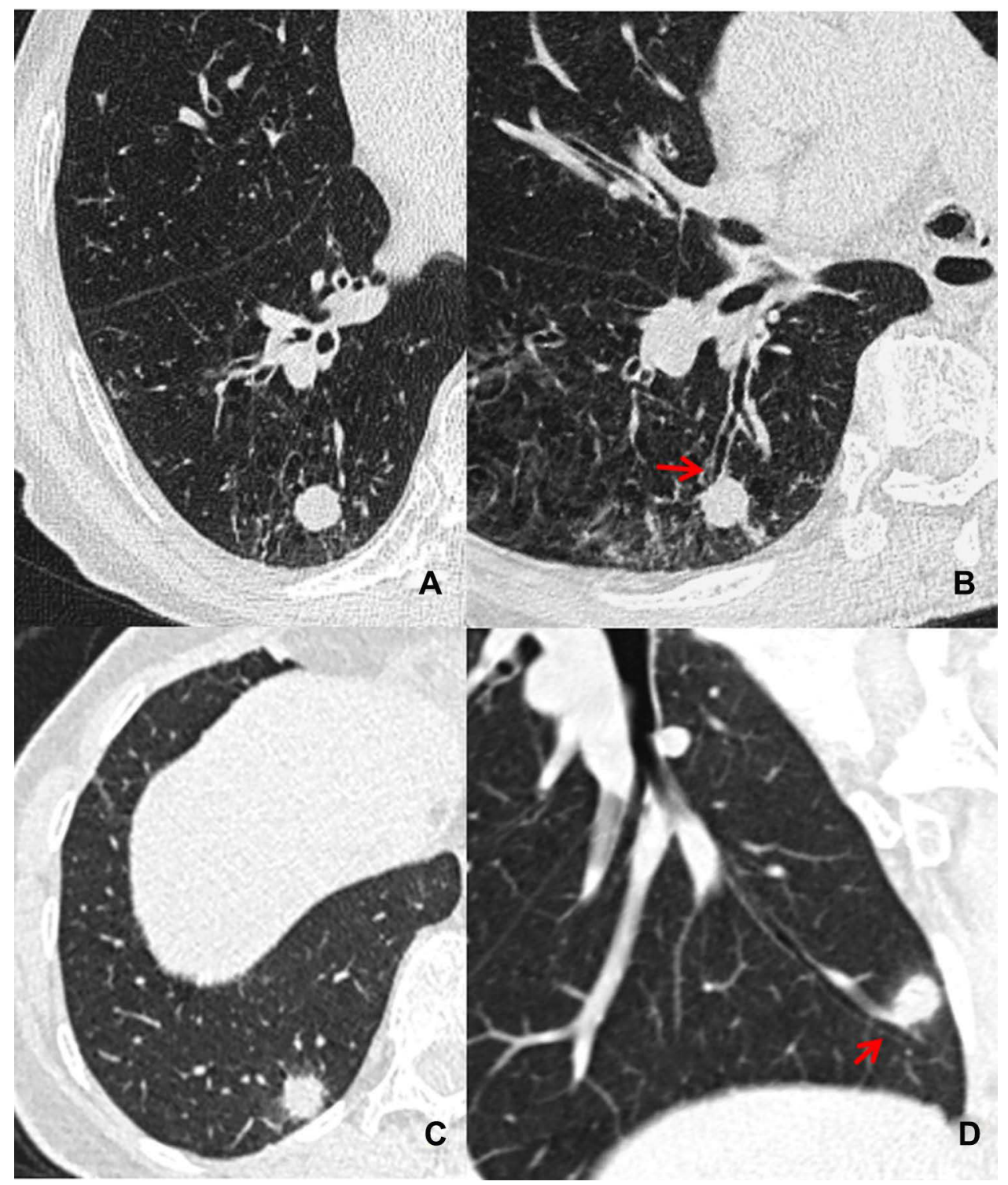

Figure 4 Differentiation between PSCLC and inflammatory lesions (pIL). Axial and reconstructed CT images in a 78 year old male with pSCLC show a round nodule with smooth margin, homogeneous density (A), and the bronchial cutoff sign (red arrow) is also detected (B). Axial and reconstructed CT images in a 46 year old female with plL show a round nodule with spiculation (C) and peripheral GGO (D) but without bronchial cutoff sign (D).

prognosis. After comparisons, it was found that pSCLCs and other nodules had some differences in their clinical characteristics and CT features. Among them, the CT morphological features of pSCLC were most similar to those of pBLT but were significantly different from those of pNSCLC and pIL. Additionally, other signs detected on CT images, such as bronchial cutoff sign, hilar lymph node enlargement, and emphysema, and some clinical characteristics were confirmed to be helpful for their differentiation. Thus, early and accurate diagnosis of pSCLC by CT examination is possible, which relies on a combination of clinical and imaging characteristics.

In this study, the bronchial cutoff sign of pSCLC was significantly higher than those of pNSCLCs, pBLTs, and pILs. The possible reason is attributed to the fact that pSCLC usually originated from mucosa and grew along submucosa layers. ${ }^{11,24}$ Thus, it was easily invaded along with the alveolar structure and peripheral bronchiole. ${ }^{25}$ At present, no other publication has reported this sign in pSCLCs in detail. The present study revealed that the type I bronchial cutoff sign was only detected in pSCLCs, and types II, III, and IV were more common in pSCLCs, pNSCLCs, and pBLTs, respectively. Therefore, the type I bronchial cutoff sign can be observed as a specific and reliable sign in diagnosing 


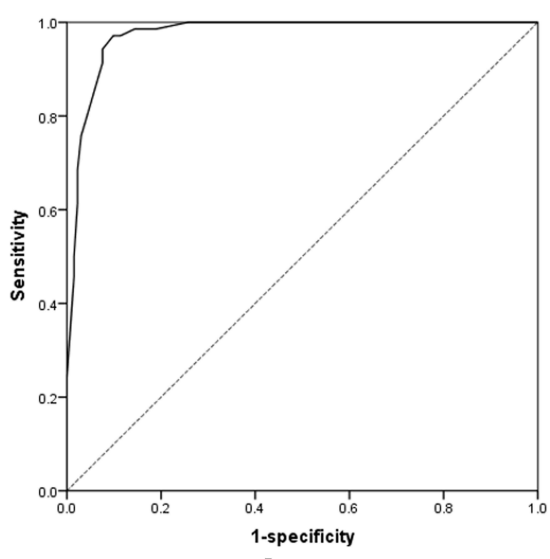

A

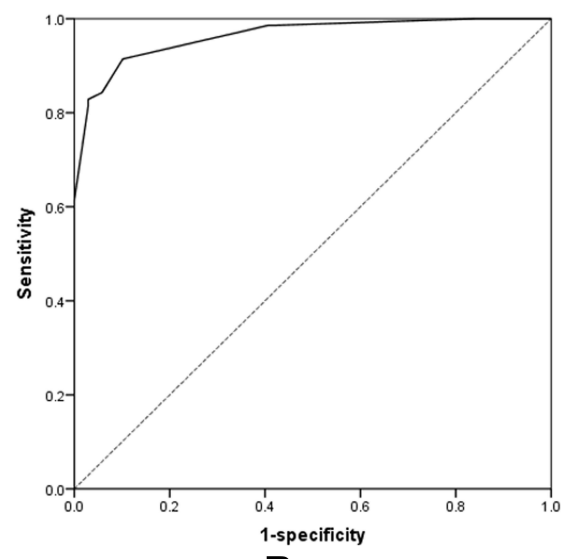

B

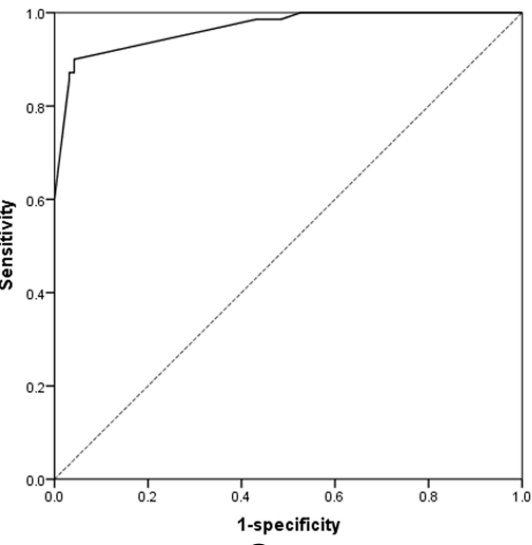

C

Figure 5 Receiver operating characteristic curves of the PSCLC predictive model established by the independent clinical and CT characteristics mentioned above. (A) performance of the pSCLC predictive model from pNSCLC; (B) performance of the pSCLC predictive model from pBLT; (C) performance of the pSCLC predictive model from plL.

pSCLCs. Type II and III bronchial cutoff sign indicated that the tumors invaded the bronchi. Thus, their presence can be considered as an indicator of malignant tumors rather than benign lesions.

Hilar or mediastinal lymph node enlargement is frequently caused by the metastasis of pulmonary malignant tumors, and it was also found to be a significant CT feature of pSCLCs in this study. For SCLCs, the tumor usually grows along the small bronchial wall or the bronchial artery and can easily invade the lung parenchyma. This may be related to the local progression of the tumor, including the hilar and mediastinal lymph node enlargement. ${ }^{8}$ Therefore, the hilar and mediastinal lymph nodes should be evaluated when a peripheral solid nodule was detected on CT images. If hilar and mediastinal lymph node enlargement were detected at the same time, the possibility of malignancy, especially SCLCs, should be suspected. In view of the benign behaviors of BLTs and ILs, the SCLCs and NSCLCs should be further differentiated on the basis of other $\mathrm{CT}$ features.

Compared with that in pNSCLC and pIL, the spiculation was less common (4.3\%) in pSCLC, which was consistent with the previous results, but the incidence was lower than them. ${ }^{8,22,26}$ In pNSCLCs and ILs, both tumor cells and inflammatory cells could infiltrate in the surrounding pulmonary parenchyma and cause interstitial thickening. However, peripheral infiltration was not significant in small-sized pSCLCs owing to the pathological basis associated with the lack of fibrous tissue. ${ }^{27}$ In addition, the vessel convergence sign and pleural indentation were also uncommon in SCLCs owing to the weak influence on the surrounding structure; these findings were consistent with previous result. ${ }^{18}$ In addition, owing to inflammatory cell infiltration, exudation, congestive edema, hemorrhage, or intra-alveolar tumor invasion, ${ }^{17}$ the peripheral GGOs in pNSCLCs and pILs were significantly higher than those in pSCLCs and pBLTs, especially in ILs. These findings are in agreement with those reported in a previously published study. ${ }^{8}$ Therefore, spiculation, vessel convergence sign, pleural indentation, and peripheral GGO can be used as important indicators for distinguishing pSCLC from pNSCLC and pIL. If a nodule demonstrated these signs, the SCLC was not considered firstly.

A previous study indicated that fusiform, vermiform, branching, and pine-cone-like shapes were the characteristics of pSCLCs. ${ }^{1,8}$ These may be related to tumor extension within the bronchi, bronchioles, or along the bronchovascular bundle, and the limitations of surrounding tissues. ${ }^{8}$ In this study, $28.6 \%$ pSCLCs had irregular shapes. This percentage was higher than those of pNSCLCs, pBLTs, and pILs. Among the irregular pSCLCs, $80 \%$ presented with shapes as those mentioned earlier. These findings were consistent with previous reports. ${ }^{8,18}$ Thus, pSCLC should be the preferred consideration when an irregular nodule with those shapes described above is detected.

SCLC is known to be strongly associated with smoking history, that is also a major cause of COPD or emphysema. ${ }^{28,29}$ A previous study showed a significantly higher risk of SCLC and squamous cell carcinoma in the presence of CT-detected emphysema than in other histological types. ${ }^{30}$ In this study, smokers and emphysema patients were more common in the pSCLC group. The percentage of smokers in the SCLC group was as high as $71.4 \%$, and was 
identical to the previous findings. ${ }^{11,16}$ In addition, males also accounted for the vast majority (80\%) of patients in the pSCLC group in this study. These findings may be related to the fact that a high-proportion of men smoke. Regarding tumor markers, proGRP in the pSCLC groups were significantly higher than those in other groups; NSE in the pSCLC groups were significantly higher than those in pNSCLC and pIL groups. This finding was also confirmed in a previous study. ${ }^{31}$ Thus, when the imaging signs are not sufficient, the gender, smoking history, and tumor markers may be useful for differentiation.

The doubling time of SCLC was relatively short. In the present study, the median doubling time was 78.5 with a range of 37-215 days derived from 10 cases, which was similar to previous results. ${ }^{1}$ Thus, the follow-up interval for the suspected pSCLC should not be too long; 1 or 2 months may be proper.

\section{Limitations}

The present study is associated with several limitations. First, it was a retrospective study and some bias may have existed. Second, the sample sizes of pSCLCs and pBLTs were relatively small. Third, because of the retrospective study, some of cases did not have complete tumor markers and some cases did not have enhanced CT data. Whether these could provide more information for the differential diagnosis should be verified in new studies.

\section{Conclusions}

In conclusion, pSCLC, pNSCLC, pBLT, and pIL have some similar and different clinical and CT features. For nodules with spiculation, pNSCLC and pIL should be considered firstly. Even though pSCLC and pBLT have similar morphological features, the bronchial cutoff sign and hilar lymph node enlargement are of great significance for differentiating them. For peripheral solid nodules, those with homogeneous density, bronchial cutoff sign, hilar lymph node enlargement, but without spiculation or vessel convergence in male patients with emphysema should be highly suspected of pSCLC, and short-term follow-up is necessary.

\section{Abbreviations}

SCLC, Small cell lung cancer; NSCLC, Non-small cell lung cancer; BLT, Benign lung tumor; IL, Inflammatory lesion; pSCLC, Peripheral small cell lung cancer; pNSCLC, Peripheral non-small cell lung cancer; pBLT, Peripheral benign lung tumor; pIL, Peripheral inflammatory lesion; OR, Odds ratio; CI, Confidence interval; PACS, Picture archiving and communication system; GGO, Ground glass opacity; Cyfra 21-1, Cytokeratin 19 fragment antigen 21-1; proGRP, Progastrin-releasing peptide; SCC-Ag, Squamous cell carcinoma antigen; CEA, Carcinoembryonic antigen; NSE, Neuron-specific enolase.

\section{Data Sharing Statement}

The datasets generated and or analyzed during the current study are not publicly available because the cases are from the Picture Archiving and Communication System of our Hospital but are available from the corresponding author on reasonable request.

\section{Ethics Approval and Consent to Participate}

The study was conducted in accordance with the Declaration of Helsinki, and the protocol was approved by the Ethics Committee of the First Affiliated Hospital of Chongqing Medical University (No. 2019-062), which absolved the need for written informed consent because of the retrospective study. All personal identification data were anonymized and deidentified before analysis.

\section{Author Contributions}

All authors made a significant contribution to the work reported, whether that is in the conception, study design, execution, acquisition of data, analysis and interpretation, or in all these areas; took part in drafting, revising or critically reviewing the article; gave final approval of the version to be published; have agreed on the journal to which the article has been submitted; and agree to be accountable for all aspects of the work. 


\section{Funding}

This work was supported by the Joint Project Chongqing Science and Technology Commission and Chongqing Public Health Commission (2022MSXM050).

\section{Disclosure}

The authors declare that they have no conflicts of interest for this work.

\section{References}

1. Sone S, Nakayama T, Honda T, et al. CT findings of early-stage small cell lung cancer in a low-dose CT screening programme. Lung Cancer. 2007;56(2):207-215. doi:10.1016/j.lungcan.2006.12.014

2. Carter BW, Glisson BS, Truong MT, Erasmus JJ. Small cell lung carcinoma: staging, imaging, and treatment considerations. Radiographics. 2014;34(6):1707-1721. doi:10.1148/rg.346140178

3. Bray F, Ferlay J, Laversanne M, et al. Cancer incidence in five continents: inclusion criteria, highlights from volume $\mathrm{X}$ and the global status of cancer registration. Int J Cancer. 2015;137(9):2060-2071. doi:10.1002/ijc.29670

4. Kalemkerian GP, Akerley W, Bogner P, et al. Small cell lung cancer. J Natl Compr Canc Netw. 2013;11(1):78-98. doi:10.6004/jnccn.2013.0011

5. Früh M, De Ruysscher D, Popat S, et al. Small-cell lung cancer (SCLC): ESMO clinical practice guidelines for diagnosis, treatment and follow-up. Ann Oncol. 2013;24(Suppl 6):vi99-105. doi:10.1093/annonc/mdt178

6. Jänne PA, Freidlin B, Saxman S, et al. Twenty-five years of clinical research for patients with limited-stage small cell lung carcinoma in North America. Cancer. 2002;95(7):1528-1538. doi:10.1002/cncr.10841

7. Weksler B, Nason KS, Shende M, Landreneau RJ, Pennathur A. Surgical resection should be considered for stage I and II small cell carcinoma of the lung. Ann Thorac Surg. 2012;94(3):889-893. doi:10.1016/j.athoracsur.2012.01.015

8. Kobayashi T, Tanaka N, Matsumoto T, et al. HRCT findings of small cell lung cancer measuring $30 \mathrm{~mm}$ or less located in the peripheral lung. Jpn J Radiol. 2015;33(2):67-75. doi:10.1007/s11604-014-0381-2

9. Rosado-de-christenson ML, Templeton PA, Moran CA. Bronchogenic carcinoma: radiologic-pathologic correlation. Radiographics. 1994;14 (2):429-446. doi:10.1148/radiographics.14.2.8190965

10. Chong S, Lee KS, Chung MJ, Han J, Kwon OJ, Kim TS. Neuroendocrine tumors of the lung: clinical, pathologic, and imaging findings. Radiographics. 2006;26(1):41-57. doi:10.1148/rg.261055057

11. Lee D, Rho JY, Kang S, Yoo KJ, Choi HJ. CT findings of small cell lung carcinoma: can recognizable features be found? Medicine (Baltimore). 2016;95(47):e5426. doi:10.1097/MD.0000000000005426

12. Kazawa N, Kitaichi M, Hiraoka M, et al. Small cell lung carcinoma: eight types of extension and spread on computed tomography. J Comput Assist Tomogr. 2006;30(4):653-661. doi:10.1097/00004728-200607000-00017

13. Suduł T, Domagała-Kulawik J. Clinical manifestation and radiological features of small cell lung cancer (SCLC). Wiad Lek. 2012;65(2):97-101.

14. Hashimoto M, Heianna J, Okane K, Hirano Y, Watarai J. Small cell carcinoma of the lung: CT findings of parenchymal lesions. Radiat Med. 1999;17(6):417-421.

15. Gustafsson BI, Kidd M, Chan A, Malfertheiner MV, Modlin IM. Bronchopulmonary neuroendocrine tumors. Cancer. 2008;113(1):5-21. doi: $10.1002 /$ cncr. 23542

16. Toyokawa G, Kozuma Y, Matsubara T, et al. Radiological features of the surgically resected small-sized small-cell lung cancer on computed tomography. Anticancer Res. 2017;37(2):877-881. doi:10.21873/anticanres.11393

17. Yabuuchi H, Murayama S, Sakai S, et al. Resected peripheral small cell carcinoma of the lung: computed tomographic-histologic correlation. J Thorac Imaging. 1999;14(2):105-108. doi:10.1097/00005382-199904000-00007

18. Ren Y, Cao Y, Hu W, Wei X, Shen X. Diagnostic accuracy of computed tomography imaging for the detection of differences between peripheral small cell lung cancer and peripheral non-small cell lung cancer. Int J Clin Oncol. 2017;22(5):865-871. doi:10.1007/s10147-017-1131-0

19. Fan L, Liu SY, Li QC, Yu H, Xiao XS. Pulmonary malignant focal ground-glass opacity nodules and solid nodules of $3 \mathrm{~cm}$ or less: comparison of multi-detector CT features. J Med Imaging Radiat Oncol. 2011;55(3):279-285. doi:10.1111/j.1754-9485.2011.02265.x

20. Shi Z, Wang Y, He X. Differential diagnosis of solitary pulmonary nodules with dual-source spiral computed tomography. Exp Ther Med. 2016;12 (3):1750-1754. doi:10.3892/etm.2016.3528

21. Li F, Sone S, Abe H, Macmahon H, Doi K. Malignant versus benign nodules at CT screening for lung cancer: comparison of thin-section CT findings. Radiology. 2004;233(3):793-798. doi:10.1148/radiol.2333031018

22. Qiang JW, Zhou KR, Lu G, et al. The relationship between solitary pulmonary nodules and bronchi: multi-slice CT-pathological correlation. Clin Radiol. 2004;59(12):1121-1127. doi:10.1016/j.crad.2004.02.018

23. Myklebust LH, Sørgaard K, Wynn R. Local psychiatric beds appear to decrease the use of involuntary admission: a case-registry study. BMC Health Serv Res. 2014;14:64. doi:10.1186/1472-6963-14-64

24. Raso MG, Bota-Rabassedas N, Wistuba II. Pathology and classification of SCLC. Cancers (Basel). 2021;13(4):820. doi:10.3390/cancers13040820

25. Guvenc C, Yserbyt J, Testelmans D, et al. Computed tomography characteristics predictive for radial EBUS-miniprobe-guided diagnosis of pulmonary lesions. $J$ Thorac Oncol. 2015;10(3):472-478. doi:10.1097/JTO.0000000000000410

26. Hashimoto M, Miyauchi T, Heianna J, et al. Accurate diagnosis of peripheral small cell lung cancer with computed tomography. Tohoku J Exp Med. 2009;217(3):217-221. doi:10.1620/tjem.217.217

27. Travis WD, Brambilla E, Nicholson AG, et al. The 2015 World Health Organization classification of lung tumors: impact of genetic, clinical and radiologic advances since the 2004 classification. J Thorac Oncol. 2015;10(9):1243-1260. doi:10.1097/JTO.0000000000000630

28. Rudin CM, Brambilla E, Faivre-Finn C, Sage J. Small-cell lung cancer. Nat Rev Dis Primers. 2021;7(1):3. doi:10.1038/s41572-020-00235-0

29. Jackman DM, Johnson BE. Small-cell lung cancer. Lancet. 2005;366(9494):1385-1396. doi:10.1016/S0140-6736(05)67569-1 
30. Li Y, Swensen SJ, Karabekmez LG, et al. Effect of emphysema on lung cancer risk in smokers: a computed tomography-based assessment. Cancer Prev Res (Phila). 2011;4(1):43-50. doi:10.1158/1940-6207.CAPR-10-0151

31. Du J, Li Y, Wang L, et al. Selective application of neuroendocrine markers in the diagnosis and treatment of small cell lung cancer. Clin Chim Acta. 2020;509:295-303. doi:10.1016/j.cca.2020.06.037

\section{Publish your work in this journal}

Cancer Management and Research is an international, peer-reviewed open access journal focusing on cancer research and the optimal use of preventative and integrated treatment interventions to achieve improved outcomes, enhanced survival and quality of life for the cancer patient. The manuscript management system is completely online and includes a very quick and fair peer-review system, which is all easy to use. Visit http://www.dovepress.com/testimonials.php to read real quotes from published authors.

Submit your manuscript here: https://www.dovepress.com/cancer-management-and-research-journal 